И зотретиноин — 40 лет в дерматологии

Самцов А.B.

ФГБВОУ ВО «Военно-медицинская академия им. С.М.Кирова» Министерства обороны Российской Федерации 194044, Россия, г. Санкт-Петербург, ул. Академика Лебедева, д. 6

В статье приводится обзор литературы, посвященный изотретиноину (Изотретиноин Lidose), который почти 40 лет является наиболее эффективным средством лечения среднетяжелых форм акне. В статье излагаются современные представления о механизме действия препарата, об эволюции взглядов на выбор дозы изотретиноина и определение длительности лечения, а также на эволюцию алгоритмов обследования больных, направленных на снижение возможных рисков развития нежелательных явлений. Приводятся данные об эффрективности и безопасности изотретиноина при акне и других дерматозах.

Ключевые слова: изотретиноин, Изотретиноин Lidose, эффективность, безопасность.

Конфрликт интересов: автор заявляет об отсутствии конфликта интересов по данной публикации.

Для цитирования: Самцов А.В. Изотретиноин - 40 лет в дерматологии. Вестник дерматологии и венерологии. 2020; 97 (2): 58—63. https://doi.org/10.25208/vdv1135 


\title{
Isotretinoin — 40 Years in Dermatology
}

\author{
Aleksey V. Samtsov
}

Federal state budgetary educational institution of higher military education "Military Medical Academy named after S.M. Kirov" of the Russian Ministry of Defense

Akademika Lebedeva str., bldg 6, Saint Petersburg, 194044, Russian Federation

The article provides a review of the literature on isotretinoin (Isotretinoin Lidose), which for almost 40 years has been the most effective treatment for moderate forms of acne. The article sets out modern ideas about the mechanism of action of the drug, as well as the evolution of treatment algorithms, as well as the evolution of algorithms for influencing people, which reduces the risk of adverse events. Data on the efficacy and safety of isotretinoin for acne and other dermatoses are provided.

Keywords: isotretinoin, Isotretinoin Lidose, efficacy, safety.

Conflict of interest: the author state that there is no potential conflict of interest requiring disclosure in this article.

For citation: Aleksey V. Samtsov. Isotretinoin - 40 Years in Dermatology. Vestnik Dermatologii i Venerologii. 2020 ; 97

(2): 58-63. https://doi.org/10.25208/vdv1135 
Оральный изотретиноин (изомер ретиноевой кислоты) около 40 лет широко применяется в лечении больных среднетяжелыми формами акне. В начале 80-х годов прошлого века началось производство изотретиноина для приема внутрь, а в 1990 г. препарат был зарегистрирован в нашей стране (капсулы 20 мг и 10 мг). В 2001 г. запатентована технология "Lidose®”, в 2009 г. в России зарегистрировали изотретиноин-Lidose (акнекутан).

В 1982 г. известный дерматолог J. Strauss написал: «Появление в дерматологической практике изотретиноина для лечения акне по своей значимости сопоставимо с внедрением топических стероидов» [1]. Его слова оказались пророческими, поскольку до настоящего времени изотретиноин является самым эффективным средством для лечения среднетяжелых фрорм акне. Это подтверждено многочисленными работами и исследованиями. В последнем руководстве Европейской академии дерматовенерологии (ЕАД) по лечению акне отмечено, что изотретиноин - наиболее эффективное средство терапии тяжелых фрорм акне [2]. H. Gollnick считает, что это единственный препарат, который прицельно воздействует на все звенья патогенеза акне [3].

В настоящее время общепризнанно, что в патогенезе акне ведущую роль в формировании микрокомедона играют следующие звенья: гиперсекреция сальных желез, фолликулярный гиперкератоз, пролиферация C.acnes/P.acnes, воспаление.

Первоначально было установлено, что первичное действие изотретиноина связано с угнетением функции сальных желез и антикератинизирующим эффректом, вторичное - с антибактериальным и противовоспалительным. Причем изотретиноин наиболее активно, по сравнению с другими препаратами, угнетает функцию сальных желез, нарушая дифференцировку себоцитов, а также влияет на способность фолликулярных кератиноцитов к сцеплению, препятствуя тем самым образованию микрокомедонов. Препарат достаточно быстро нормализует фолликулярную кератинизацию, что приводит к уменьшению колонизации C.acnes/P.acnes и воспалительных явлений. В последние годы выявлены новые механизмы действия, объясняющие клинический эффрект изотретиноина.

В частности, было показано, что изотретиноин обладает локальным антиандрогенным действием за счет конкурентного ингибирования энзимов ретинолдегидрогеназ, ответственных за оксидацию андрогенов в себоцитах [4]. Кроме того, выявлено продолжительное действие изотретиноина на факторы врожденного иммунитета и воспаление, в частности, за счет уменьшения экспрессии толл-подобных рецепторов-2 (TLR-2) на моноцитах у пациентов с тяжелыми акне. Было также установлено пролонгированное воздействие на TLR-2 в течение 6 месяцев после прекращения системной терапии, что объясняет клиническое выздоровление или стойкое улучшение у таких больных, а также развитие рецидивов через 6 месяцев после отмены препарата [5]. Доказано, что изотретиноин снижает активность матриксных металлопротеиназ-1, -3, -13, играющих роль в фрормировании рубцов, и повышает активность тканевых ингибиторов указанных протеиназ [6], что объясняет во многом эффрект в отношении рубцовых изменений. Кроме того, изотретиноин воздействует на биоплен- ку, снижая тем самым риск развития резистентности, приводит к нормализации реакции врожденного иммунитета, а совсем недавно установили положительное влияние на микробиом [7].

Изначально изотретиноин был рекомендован для лечения тяжелых форм акне, однако в дальнейшем, с учетом накопленного опыта по безопасности препарата, показания расширили, включив акне средней степени тяжести. Согласно последним руководствам Американской академии дерматологии (ААД) и Европейской академии дерматовенерологии, показания для назначения изотретиноина следующие: тяжелая степень папуло-пустулезных акне/умеренная узловатых, узловатые/ конглобатные акне, акне, резистентные к традиционной терапии, быстрый рецидив после отмены системных антибиотиков, психосоциальные расстройства по поводу акне, склонность к заживлению акне с образованием рубцов [2, 8]. В руководстве Европейская академия дерматовенерологии уровень доказательности монотерапии изотретиноином определяется как высокий [2], а в Американская академия дерматологии препарат относит к первой линии терапии акне [8].

Исследования последних лет свидетельствуют о неослабевающем интересе к изотретиноину, что подтверждают новые работы по повышению его эффективности. В частности, H. Gollnik и соавт. рекомендуют раннее назначение изотретиноина при наличии как минимум 2 пунктов в следующих случаях: указание на акне в семейном анамнезе, начало заболевания в раннем возрасте, гиперсеборея, локализация на туловище, развитие рубцов, психоэмоциональная лабильность, поздние акне [3].

Ключевыми вопросами при лечении изотретиноином являются выбор дозы и длительности терапии. Согласно последним рекомендациям Американской академии дерматологии, изотретиноин следует назначать 0,5 мг/кг в первый месяц, затем постепенно увеличивать дозу до 1,0 мг/кг 2 раза в день. Длительность лечения составляет 15-20 недель. При средней степени тяжести акне рекомендованы дозы из расчета 0,25-0,4 мг/кг. В этих случаях оправданны более низкие кумулятивные дозы [8]. Согласно рекомендациям Европейской академии дерматовенерологии, при тяжелой степени папуло-пустулезных акне/умеренной узловатых следует назначать 0,3-0,5 мг/кг, при конглобатных акне - 0,5 мг/кг и более. При этом длительность терапии составляет не менее 6 месяцев, а при недостаточной эффективности показано ее продолжение [2]. Таким образом, отмечается четкая тенденция к удлинению сроков терапии с 12 недель, предложенных в первых рекомендациях, до 20 недель в рекомендациях 2007 г. [9] и до 24 недель в рекомендациях 2016 г. [2, 8]. По последним данным D. Thiboutot и соавт., терапия изотретиноином должна проводиться до полного или почти полного очищения кожи [10]. В случае плохой реакции на лечение следует учитывать такие факторы, как стресс, диета, Malassezia furfur, G-отрицательные бактерии, комедогенные средства по уходу за кожей, эндокринные расстройства [3].

Данные о рецидивах заболевания весьма противоречивы, так, H. Gollnick и соавт. сообщают о 25-30\% случаев рецидивов после 1-го курса терапии [3], в то время как Brzezinski и соавт., наблюдавшие 3525 больных, - всего лишь о 1,67\% [11]. J. Tan счи- 
тает, что вероятность рецидива заболевания при применении низких доз значительно возрастает, и это требует более продолжительного курса лечения, чем при использовании стандартных схем [12]. Согласно руководству ААД, рецидивы реже встречаются при кумулятивной дозе свыше 120 мг/кг, чем при дозе менее 120 мг/кг, оптимальными дозами при тяжелых фрормах являются 120-150 мг/кг, а $220 \mathrm{мг/кг} \mathrm{вызывает} \mathrm{еще}$ меньше рецидивов [8]. Последние исследования показали, что дозы изотретиноина свыше 200 мг наиболее эфффективны в отношении рецидивов [13].

Одним из важнейших вопросов при лечении изотретиноином является вопрос о безопасности. При этом огромный опыт лечения привел к существенному изменению взглядов на алгоритм обследования больных с целью минимизации риска развития нежелательных явлений.

Следует подчеркнуть, что препарат обладает побочными действиями и противопоказан при беременности. Наиболее часто отмечаются хейлит, сухость кожи, шелушение, реже - алопеция, конъюнктивит, головная боль, артралгии. Изначально было предложено очень сложное мониторирование, в основе которого лежало регулярное исследование функции печени, липидного обмена, ряда других показателей, однако по мере накопления опыта представления о токсичности препарата менялись. В частности, В рекомендациях Американской академии дерматологии 2007 г. отмечено следующее: стандартный 20-недельный курс лечения обычно хорошо переносится и безопасен; побочные явления со стороны кожи и слизистых возникают часто, являются обратимыми, хорошо поддаются увлажняющей местной терапии и не требуют отмены препарата; побочные эффректы со стороны костей редкость; могут встречаться небольшие лабораторные отклонения, не требующие отмены препарата, следует определять лишь исходные показатели функции печени и липидного обмена и через 2-4 недели приема препарата; КППЛ (контрацептивный период после лечения) составляет 1 месяц [9]. Согласно руководству Американской академии дерматологии 2016 г., низкие дозы могут быть эфффективны и уменьшают частоту и тяжесть нежелательных явлений; интермиттирующая терапия не рекомендуется; показано исследование функции печени и липидного обмена до лечения и через 2 месяца после начала, при нормальных показателях необходимости в повторных назначениях нет; рекомендуется программа iPLEDGE (контрацепция проводится за 1 месяц до начала лечения, во время и 5 недель после с использованием двух видов контрацептивов - барьерных и гормональных с проведением ежемесячного теста на беременность на протяжении всего курса лечения); следует мониторировать воспалительные заболевания кишечника и депрессивные состояния [8].

Алгоритм, предложенный Европейской академией дерматовенерологии, несколько строже: мониторирование энзимов печени и липидов следует проводить до начала лечения, через 1 месяц, затем каждые 3 месяца; важно выполнять программу по предупреждению беременности; не применять лазеротерапию, пилинги как минимум 6 месяцев после окончания лечения [2].

Из последних работ по оценке нежелательных явлений при лечении изотретиноином заслуживают внимания данные Brzezinski и соавт., которые наблюдали в течение 5 лет 3525 больных среднетяжелыми формами акне в возрасте от 13 до 35 лет. Хейлит выявлен у $100 \%$ больных; ксероз - у 95\%; эритема лица у 66\%; психоэмоциональные нарушения - у 25\%; поражения глаз - у 9\%; повышение уровня триглицеридов - у $3 \%$; печеночных ферментов - у $2 \%$ [11]. В 2020 г. были опубликованы данные о нежелательных явлениях при лечении изотретиноином 393 женщин: хейлит и ксероз - у 97,3\% больных; носовые кровотечения - у 5,8\%; повышение уровня печеночных ферментов - у 0,8\%; нарушение липидного обмена у $0,5 \%[14]$.

Таким образом, исследования последних лет свидетельствуют о незначительной частоте нежелательных явлений, связанных с воздействием на печень и липидный обмен, при этом они были выражены незначительно и не требовали отмены препарата или снижения дозы. В 2017 г. Huang и Cheng опубликовали большой обзор литературы и метаанализ, в котором показали, что нет связи приема изотретиноина с увеличением риска депрессии, более того, лечение изотретиноином приводит к улучшению психического состояния больных акне [15].

С целью уменьшения проявлений хейлита и ретиноидного дерматита, связанных с системным изотретиноином, показано применение крема на основе эктоина (перфэктоин). Он представляет собой новый класс продуктов для длительного увлажнения кожи. В основе натуральный компонент эктоин, который обладает способностью к удержанию воды, формируя своеобразную водную мантию «эктоин-гидрокомплекс». Эктоин был выделен шведскими учеными и в креме присутствует в максимально возможной на текущий момент концентрации 7\%. Эктоин-гидрокомплекс обеспечивает длительное и выраженное увлажнение кожи и препятствует ее контакту с аллергенами и раздражающими веществами из окружающей среды. Также доказан защитный эффект от УФ-облучения, что важно при терапии изотретиноином.

H. Gollnik рекомендует снижать потенциальные побочные эффректы при общей терапии изотретиноином следующими методами: мыть лицо 1р/д мягкими очищающими средствами без мыла; наносить увлажняющие средства 2-3 р/д; использовать фротозащитные препараты с SPF 25-50 для минимизации риска развития гиперпигментных пятен и морщин; прием антигистаминных средств [3].

В 2001 г. для изотретиноина была запатентована новая форма, произведенная по технологии Lidose (Бельгия, представителем которой в РФ является препарат акнекутан). Внедрение новой формы было связано с тем, что изотретиноин относится к плохо растворимым соединениям и усваивается в пищеварительном тракте не полностью. Поэтому увеличение растворенной фрракции изотретиноина повышает его усвояемость. Технология Lidose заключается в добавлении 2 дополнительных липофильных компонентов в состав препарата (гелюцир и спан), благодаря которым увеличивается растворенная фракция изотретиноина, и изотретиноин Lidose более полно усваивается в пищеварительном тракте относительно обычной формы изотретиноина. При этом его биодоступность увеличивается на 20\%. В результате проведенных исследований было показано, что 8 мг изотретиноина Lidose (Акнекутан) эквивалентны 10 мг обычного 
изотретиноина. A 16 мг изотретиноина Lidose (акнекутан) биоэквивалентны 20 мг обычного изотретиноина.

Изотретиноин в виде капсул, содержащих жидкие (полужидкие) липидные эксципиенты, был одобрен FDA и Минздравом Канады в 2012 г. и имеет те же показания, что и изотретиноин. Система Lidose обеспечивает необходимые условия для эффрективного и быстрого всасывания заявленной в капсуле дозы лекарственного препарата в кишечнике [16]. К настоящему времени опубликован ряд исследований, демонстрирующих высокую эффрективность и безопасность терапии изотретиноином Lidose при долгосрочном применении. В масштабном двойном слепом рандомизированном контролируемом 20-недельном клиническом исследовании с участием 925 пациентов в возрасте от 12 до 54 лет с тяжелыми, рецидивирующими акне подтверждена высокая эффективность растворимой основы Lidose [16]. Изотретиноин Lidose не связан с приемом пищи, препарат можно принимать натощак [8].

В заключение следует отметить, что увеличение биодоступности позволяет снижать рекомендованные разовые и курсовые дозы при терапии изотретиноином Lidose на 20\%, по сравнению с изотретиноином в обычной форме выпуска (диапазон рекомендованных суточных доз для изотретиноина Lidose (акнекутан) составляет 0,4-0,8 мг/кг, курсовых доз - 100-120 мг/кг), что при сохранении одинаковой эффективности терапии, в итоге приводит к снижению риска развития нежелательных явлений.

Согласно инструкции, изотретиноин показан для лечения тяжелых форм акне и акне, не поддающихся другим видам терапии, однако в литературе имеются сообщения об эфффективности препарата при других дерматозах.
В частности, работы по эфрфективности изотретиноина при розацеа периодически появлялись начиная с 1980-х годов. Препарат приводил к положительному результату в дозах 0,1; 0,3; 0,5; 1 мг/кг/д или 10 мг в день в течение 3-4 месяцев и более. Van Zuuren и соавт. применяли изотретиноин при тяжелых фрормах розацеа и ринофиме, малые дозы изотретиноина (0,25 мг/кг/д) оказывали выраженный эффрект на папуло-пустулезный субтип розацеа, трудно поддающийся терапии [17]. Терапевтический эффрект при розацеа объясняется противовоспалительным, антиоксидантным, антиангиогенным и антифиибротическим действиями [18]. В 2012 г. были опубликованы результаты лечения изотретиноином Lidose (акнекутан) 21 больного папуло-пустулезным субтипом розацеа, у которых заболевание не поддавалось терапии традиционными средствами. Препарат назначали 1 раз в сутки в малых дозах - 0,2 мг/кг. Длительность терапии определялась не набором какой-либо курсовой дозы, а степенью выраженности клинического эфрфекта, и составила от 5 до 7 месяцев. Анализ полученных данных свидетельствовал о высокой эффрективности изотретиноина в лечении папуло-пустулезного подтипа розацеа [19].

В связи с вышеизложенным изотретиноин (изотретиноин Lidose) был внесен в Федеральные клинические рекомендации по ведению больных розацеа [20].

Имеются также сообщения об эфффективности изотретиноина при множественных бородавках лица (препарат нарушает диффреренцировку кератиноцитов) [21]. По мнению Tietze и соавт., изотретиноин - самое эффрективное средство для лечения декальвирующего фолликулита [22], препарат также назначают больным генерализованной кольцевидной гранулемой [23].

\section{Литература/References}

1. Strauss J. Introduction. J Am Acad Dermatol 1982; 6: 573—575.

2. Nast A., Dreno B., Bettoli V. et al. European Evidence-based (S3). Guideline for the Treatment of Acne. Update 2016. JEADV 2016, 30: 1261-1268.

3. Gollnick H., Abanmi A., Al-Enezi M. et al. Managing acne in the middle east:consensus recommendation. JEADV,2017:31 (Suppl 7): $4-35$.

4. Karlsson T., Vahlquist A., Kedishvili N., Törmä H. 13-cis-retinoic acid competitively inhibits 3 alpha-hydroxysteroid oxidation by retinol dehydrogenase RoDH-4: a mechanism for its anti-androgenic effects in sebaceous glands. Biochem Biophys Res Comm. 2003; 303: 273—278.

5. Dispenza M., Wolpert E.B., Gilliland K.L. et al. Systemic isotretinoin therapy normalizes exaggerated TLR-2-mediated innate immune responses in acne patients. J Invest Dermatol. 2012; 132: 2198-2205.

6. Lee J.W., Yoo K.H., Park K.Y. et al. Effectiveness of conventional, low-dose and intermittent oral isotretinoin in the treatment of acne: a randomized, controlled comparative study. Br J Dermatol. 2011; 164; $1369-75$.

7. Stein R. Dermatology Daily 9.01.2019.

8. Zaenglein A., Pathy A., Schlosser B. et al. Guidelines of care for the management of acne vulgaris. J Am Acad Dermatol. 2016; 74: 945-973.

9. Strauss J., Krowchuk D., Leyden J. et al. Guidelines of care for acne vulgaris management. J Am Acad Dermatol 2007; 56: 651—663.
10. Thiboutot D., Dreno B., Abanmi A. et al. Practyical management of acne for clinicians: An international consensus from the global Alliance to Improve Outcomes in Acne. Am J Acad Dermatol 2018, 78; 2: S.1-23.

11. Brzezinski et al. Adverse effects of isotretinoin. Dermatologic therapy 2017,D0l10.1111/dth.12483.

12. Tan J. A treatment for severe nodular acne. Br J Dermatol 2014; 171: $1508-1516$.

13. Zeitany A. High-dose isotretinoin has lower impact on wallets. J Am Acad Dermatol 2016; 74: 175-176.

14. Fernandes J., Briggit Lee, Patel J. et al. Retrospective case series of isotretinoin outcomes for acne in 393 female patients at Baylor College of Medicine during 2012-2016. J Am Acad Dermatol 2020; 82 : 1219-1220.

15. Huang Yu-Chen, Cheng Ying-Chih. Isotretinoin treatment for acne and risk of depression: a systematic review and meta-analysis. J Am Acad Dermatol 2017; 1068-76.

16. Laboratories S.M.B., Brussels, Belgium. Web site. Lidose. Available at: http://www.smblab.be/index.php/formulation/lidose. Accessed July 29, 2013.

17. Van Zuuren et al. Low-Dose Isotretinoin: An Option for Difficult-to-Treat Papulopustular Rosacea. Journal of Investigative Dermatology (2016) 136, 1081-1083.

18. Thielitz E. Rosacea. Systemic therapy with retinoids. Hautarzt 2011; 62: 820—827. 
19. Самцов А.В., Горбунов Ю.Г. Изотретиноин в лечении розацеа. Вестн дерматол 2012; 4: 102-104. [Samtsov A.V., Gorbunov U.G. Isotretinoine in the treatment of rosacea. Vestn Dermatol Venerol 2012; 4: 102-104 (Russia).]

20. Кубанова А.А., Кубанов А.А., Аравийская Е.А., Самцов А.В. Федеральные клинические рекомендации. Дерматовенерология 2015: Болезни кожи. Инфекции, передаваемые половым путем. 5-е изд., перераб. и доп. М.: Деловой экспресс, 2016. 768 с. [Kubanova A.A., Kubanov A.A., Araviyskaya E.A., Samtsov A.V. Federal clinical guidelines.
Dermatovenerology 2015: Skin diseases. Sexually transmitted infections. 5th ed., Revised. and add. M.: Business Express 2016. 768 p. (Russia).]

21. Kaur $Y$. et al. Evaluation of the efficacy and safety of oral isotretinoin versus topical isotretinoin in the treatment of plane warts: a randomized open.Int J Derm. doi:10.1111/ijd.13727.

22. Tietze J., Heppt M., Preusen A. Oral isotretinoin as the most effective treatment in folliculitis decalvans. JEADV 2015;8:1467.

23. Lukacs J., Schliemann S., Elsner P. Treatment of generalized granuloma annulare — a systematic review. JEADV 2015;9:1816.

\section{Информация об авторе}

Самцов Алексей Викторович - д.м.н., профрессор, заведующий кафедрой кожных и венерических болезней Военно-медицинской академии им. С.М. Кирова; тел.: +7 (812) 271-87-81, e-mail: avsamtsov@mail.ru

\section{Information about the author}

Aleksey V. Samtsov - Dr. Sci. (Med.)., Prof., Head of the Department of Skin and Sexually Transmitted Diseases, S.M. Kirov Military Medical; tel.: +7 (812) 271-87-81, e-mail: avsamtsov@mail.ru 